\title{
Avaliação da Taxa de Resposta à Terapia de Ressincronização: o Super-Respondedor
}

Evaluation of Response Rate to Resynchronization Therapy: the Super-Responder

Izaias Marques de Sa Junior ${ }^{1, *}$, Jose Carlos Pachón Mateos ${ }^{1,2}$, Juan Carlos Pachón Mateos ${ }^{1,2}$, Remy Nelson Albornoz Vargas ${ }^{1,2}$

ORCID IDS

Sa Junior IM (iD https://orcid.org/0000-0002-6689-4102

\section{RESUMO}

A terapia de ressincronização cardíaca (TRC) surgiu como modalidade terapêutica para pacientes com insuficiência cardíaca (IC) refratária ao tratamento farmacológico. Ao longo dos últimos 20 anos, vários estudos clínicos buscaram estabelecer seus benefícios em diferentes populações. A revisão dos resultados desses estudos demonstrou que em pacientes com IC avançada [classes funcionais (CFs) I, II, III e IV da New York Heart Association (NYHA)] a TRC produz melhorias consistentes para a qualidade de vida, CF e capacidade de exercício, além de reduzir as hospitalizações e a taxa de mortalidade. Até 70\% dos pacientes submetidos à TRC evoluem como respondedores. Os critérios adotados na avaliação da taxa de resposta à TRC serão elucidados neste artigo, no qual o objetivo maior é ressaltar o conceito do super-respondedor à TRC.

PALAVRAS-CHAVE: Terapia de ressincronização cardíaca; Respondedor; Super-repondedores.

\begin{abstract}
Cardiac resynchronization therapy (CRT) emerged as a therapeutic modality for patients with cardiac insufficiency $(\mathrm{Cl})$ refractory to pharmacological treatment. Over the last 20 years, several clinical studies have sought to establish their benefits in different populations. The review of the results of these studies has shown that in patients with advanced CI (functional class (FC) I, II, III and IV of the New York Heart Association (NYHA) CRT produces consistent improvements in quality of life, FC and exercise capacity, as well as reducing hospitalizations and mortality rates. Up to $70 \%$ of patients submitted to CRT evolve as responders. The criteria adopted in the evaluation of the CRT response rate will be elucidated in this article, in which the main objective is to highlight the concept of the CRT super-responder.
\end{abstract}

KEYWORDS: Cardiac resynchronization therapy; Respondent; Super-responders.

1. Instituto Dante Pazzanese de Cardiologia - São Paulo/SP - Brasil.

2.Associação do Sanatório Sírio - Hospital do Coração - Setor de Eletrofisiologia - São Paulo/SP - Brasil.

*Autor correspondente: izaiasdesa@gmail.com

Recebido: 24 Out 2016 | Aceito: 07 Jul 2017

Editor Associado: Genildo Ferreira Nunes 


\section{INTRODUÇÃO}

A insuficiência cardíaca(IC) representa um grande problema para a saúde pública mundial. Na Europa e nos Estados Unidos representa importartante causa de hospitalização em pacientes com idade igual ou superior a 65 anos $^{1,2}$. Essa doença tem grande impacto social, econômico e, sobretudo, humano, pois impõe importante limitação física aos pacientes, resultando em aposentadorias precoces, com altos custos governamentais ${ }^{3}$.

Apesar dos avanços das terapêuticas farmacológicas disponíveis, essas não atendem completamente às necessidades dos pacientes com falência cardíaca ${ }^{4}$. Terapias não farmacológicas, como os dispositivos mecânicos de assistência ventricular esquerda e o transplante cardíaco, são reservadas para um pequeno grupo de pacientes que apresenta $\mathrm{IC}^{5,6}$ Somando-se a essas, a terapia de ressincronização cardíaca (TRC) e os cardiodesfibriladores implantáveis surgiram como boa opção para os pacientes com IC refratária ao tratamento medicamentoso ${ }^{4-7}$.

A base fisiopatológica da TRC é a presença de dessincronia associada ao distúrbio de condução. Com o implante de eletrodos no átrio direito $(\mathrm{AD})$, ventrículo esquerdo (VE) e ventrículo direito (VD) e programação adequada do dispositivo alcançaríamos a ressincronização atrioventricular e ventrículo-ventricular e melhora esperada do paciente. A TRC está indicada em portadores de IC com distúrbio de condução, principalmente com morfologia de bloqueio de ramo esquerdo (BRE), classes funcionais ( $\mathrm{CFs}$ ) II, III, IV NYHA e tratamento clínico otimizado ${ }^{8,9}$.

A taxa de resposta à TRC historicamente se aproxima de $70 \%$ dos casos. Com a melhoria da seleção dos pacientes, ou seja, duração QRS > 150 ms e morfologia $\mathrm{BRE}^{10}$, ausência de fibrose pela ressonância magnética $(\mathrm{RM})$ na região posterolateral ${ }^{11}$, bem como carga de fibrose total $<20 \%{ }^{12}$, melhora da técnica cirúrgica, programação adequada do dispositivo, observamos um incremento recente e considerável na taxa de resposta à TRC

A seguir relatar-se-á o caso de um paciente considerado super-respondedor à TRC. Serão discutidos, também, quais as variáveis importantes na seleção desses pacientes, bem como os critérios para serem considerados super-respondedores.

\section{RELATO DO CASO}

MJS, 72 anos, natural do estado da Paraíba, portador de IC desde 2010, sem outras comorbidades. Admitido em um hospital terciário em 2015 para otimização e investigação da etiologia IC.

Nesse período, em CF III, otimizaram-se as doses de carvedilol, enalapril, espirinolactona e furosemida. O paciente teve melhora considerável da $\mathrm{CF}$, agora CF II. Foram feitos todos os exames necessários para elucidar a etiologia da IC (Chagas, ecocardiograma, RM do coração, cintilografia miocárdica), permanecendo, como diagnóstico definitivo, IC idiopática.

Por ser portador de BRE e ainda estar sintomático a despeito do tratamento clínico, foi encaminhado à equipe de marcapasso para decidir quanto à TRC. Com base nas atuais evidências, optou-se pelo implante do ressincronizador cardíaco, realizado 13/12/2015.

Definiram-se cinco passos essenciais para o sucesso na utilização da TRC como modalidade de tratamento. Foram seguidos rigorosamente.

\section{Seleção do paciente}

De acordo com as atuais evidências, IC com fração ejeção < 35\% e BRE com duração do QRS > $150 \mathrm{~ms}$ talvez sejam uma das únicas indicações consideradas CF IA de $\mathrm{TRC}^{8,9}$. No presente caso, esses critérios foram satisfeitos.

\section{Técnica cirúrgica}

A dessincronia cardíaca gerada pelo BRE tem como a região mais lenta, mais atrasada, a condução na parede posterolateral do $\mathrm{VE}^{13}$. Esse deve ser o local de implante do eletrodo de VE. A veia cardíaca de eleição deverá ser aquela que se dirige à região posterolateral do VE (Fig. 1). Além disso, a realização de RM do coração pré-implante descarta a presença de fibrose nessa região, evitando, assim, possível estimulação em região não viáve ${ }^{11}$. No presente caso, todos esses cuidados foram observados.

\section{Raio X e comparação dos eletroencefalogramas (ECGs) (< um mês de pós-operatório)}

Pode-se certificar da posição correta dos eletrodos, principalmente do eletrodo do seio coronário $\mathrm{VE}$, em região posterolateral (Fig. 2). Em caso de deslocamento, deverá ser reabordado cirurgicamente para reposicionamento. No presente caso, manteve-se o eletrodo VE estável no pós-operatório.

Além disso, uma redução considerável na duração QRS pré e pós-implante fornece boas perspectivas em relação a 
chances de resposta à TRC (Fig. 3). No presente caso, houve redução de $18,75 \%$ da duração QRS.

\section{Programação adequada do ressincronizador}

Deverá ser confirmada a estimulação biventricular (> 90\% captura pela telemetria, idealmente $100 \%{ }^{14}$ (Fig. 4), bem como os limiares de estimulação. Atenção especial a outros fatores que diminuem a resposta TRC, como, por exemplo, presença de fibrilação atrial e extra-sístoles ventriculares frequentes e outros associados à piora da qualidade de vida, a saber, estimulação frênica (ausentes no caso relatado).

Sugere-se o ajuste do intervalo atrioventricular (IAV) em torno de $120 \mathrm{~ms}^{15}$ (Fig. 5). A programação do intervalo de estimulação entre os ventrículos (VV) deverá ser aquela em que se produz o menor QRS na estimulação bivetrincular no ECG de 12 derivações, de forma geral, intervalo VV programado entre $0 \mathrm{~ms}$ ou $-30 \mathrm{~ms} \mathrm{VE}>\mathrm{VD}$ (Fig. 5). No presente caso, adotaram-se esses conceitos.

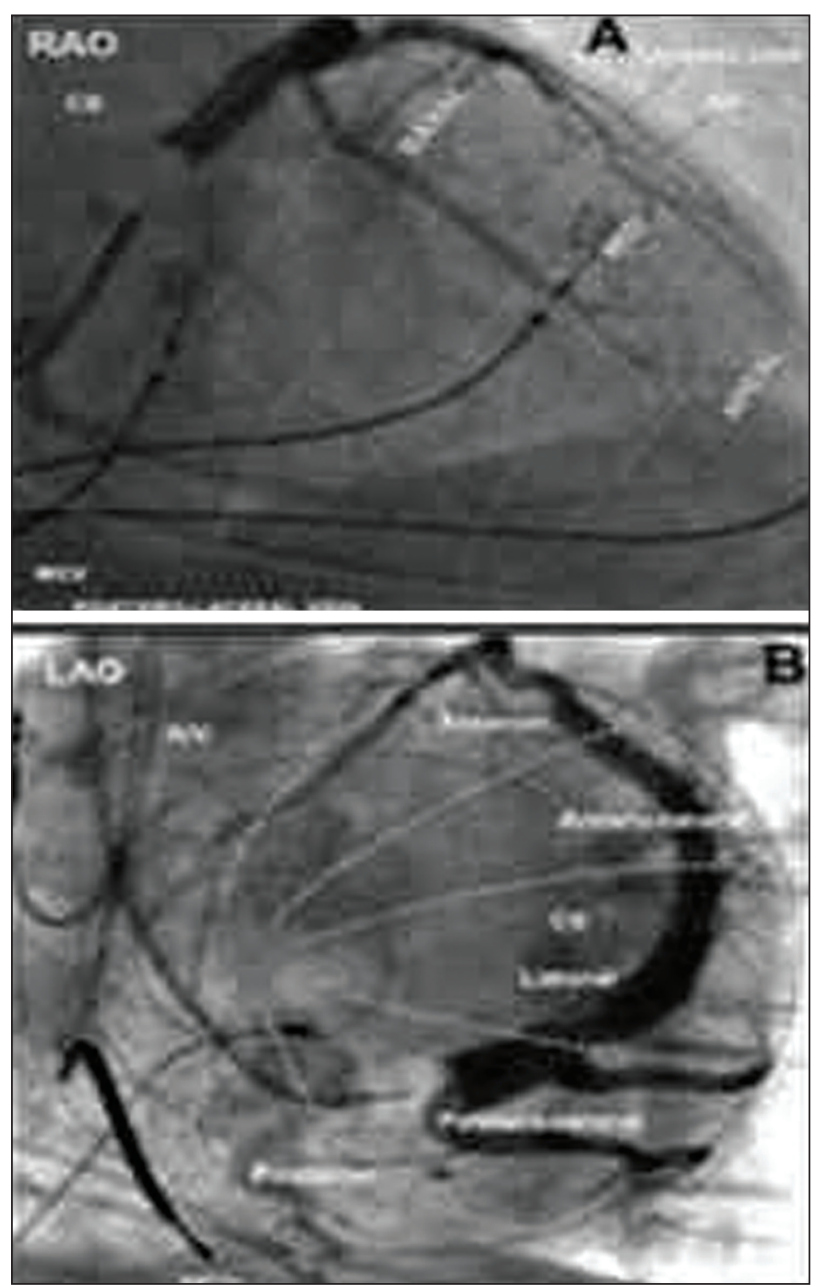

Figura 1. Anatomia seio coronário.
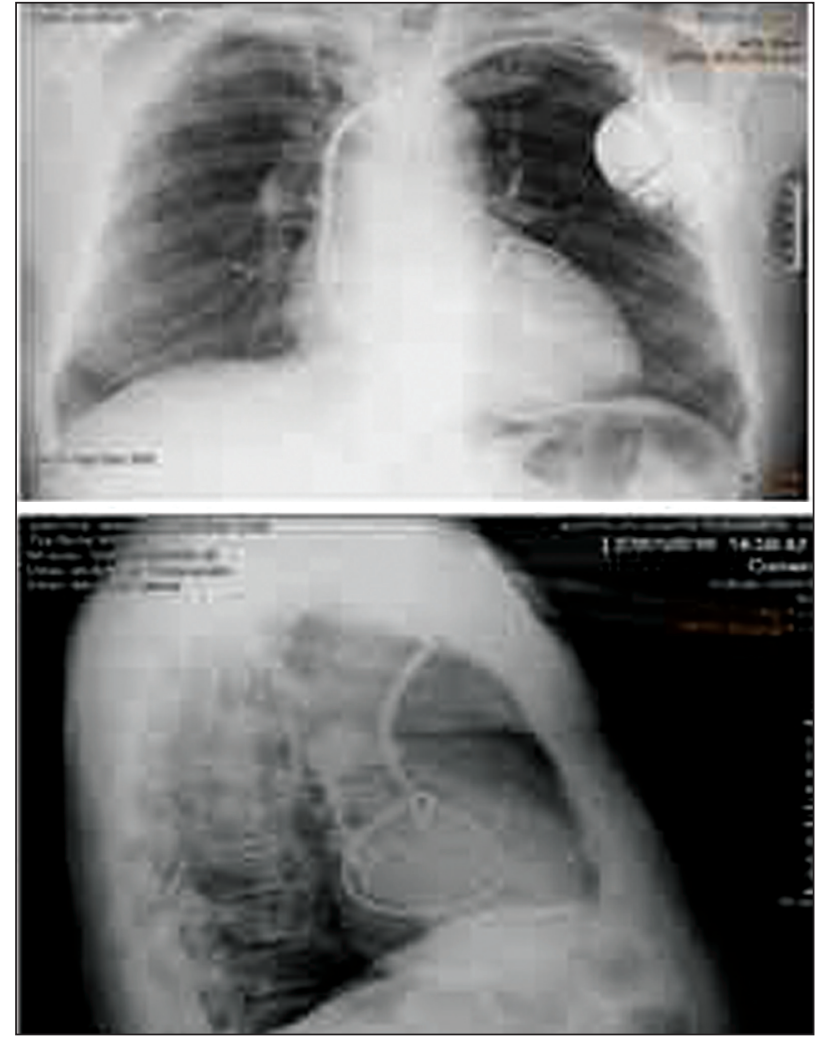

Figura 2. Posição eletrodo VE parede póstero lateral
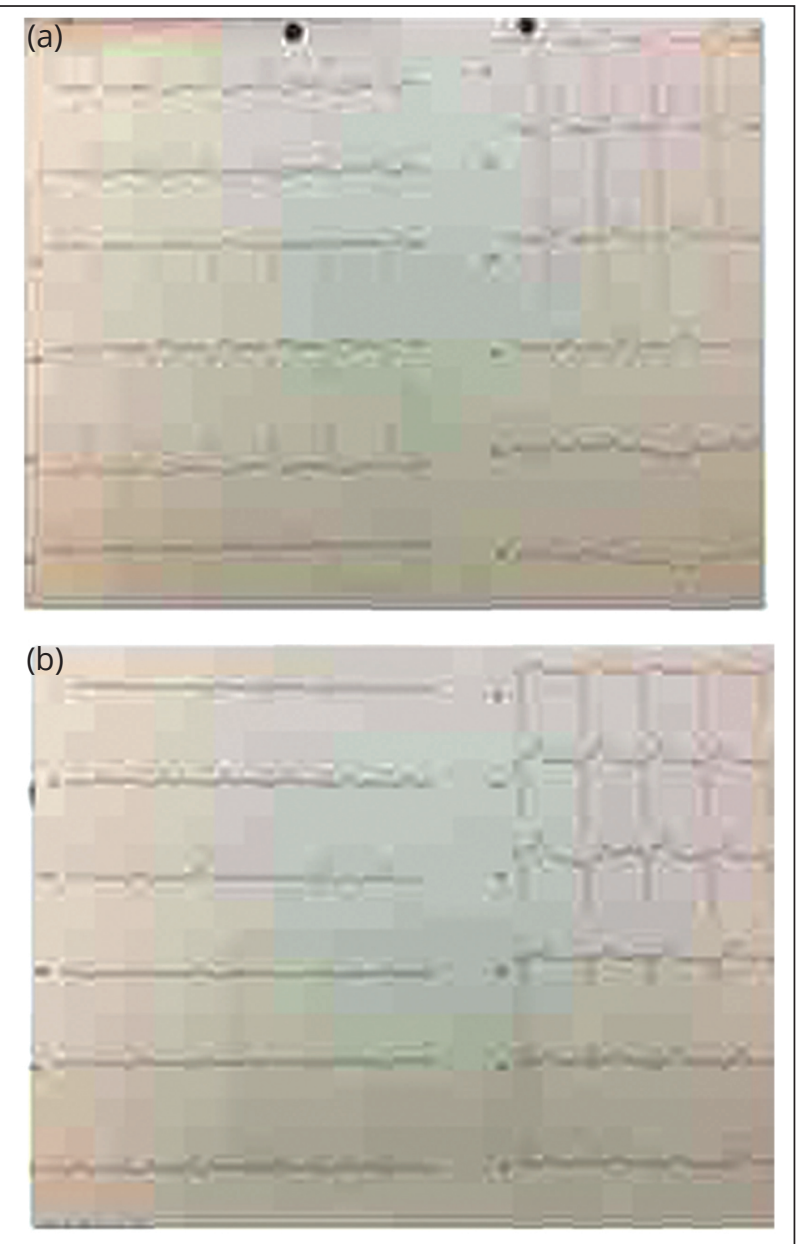

Figura 3. (a) ECG pré e (b) ECG pós impante. 


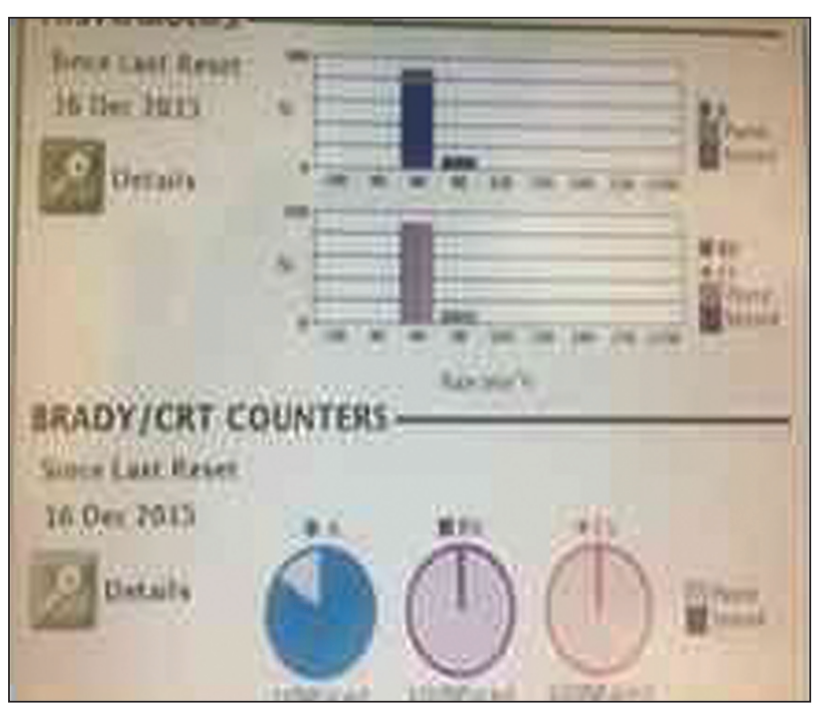

Figura 4. $100 \%$ captura de VE.

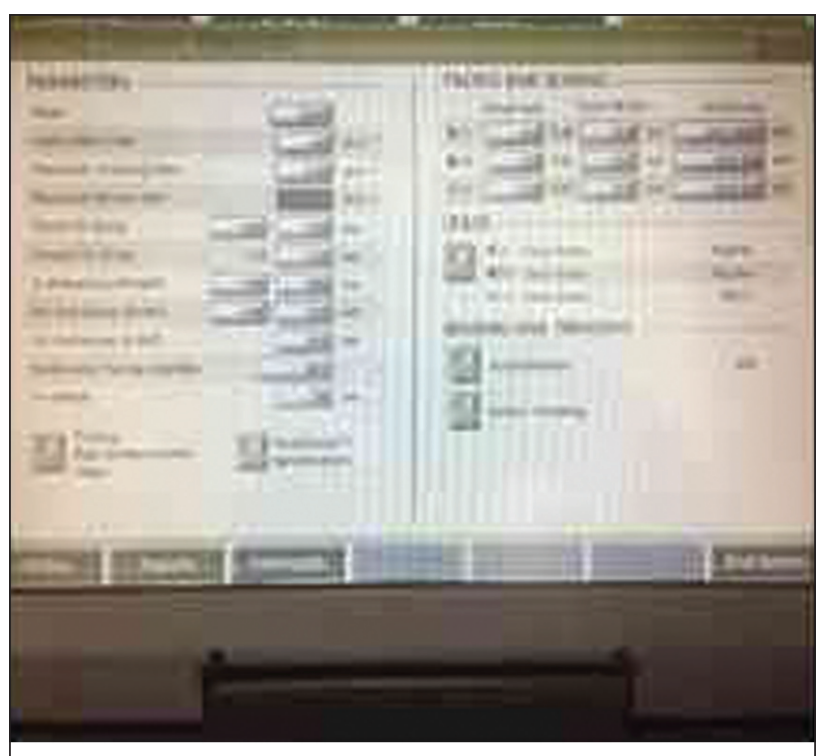

Figura 5. IAV 120MS e VV-30MS.

\section{Avaliação da taxa de resposta TRC}

Por último, avaliar-se-á se de fato nosso paciente respondeu à TRC. De acordo com os principais estudos, seis meses seria o tempo suficiente para se ter essa resposta. Por meio de critérios clínicos (CF), ecocardiográficos [função ventricular (FV), volume sistólico final (VSF)] e outros podemos concluir a respeito. No presente caso, após avaliação, concluiu-se tratar-se de um superrespondedor. Pelos dados ecocardiográficos, a FV de $28 \%$ foi para 55\% (normalização), O VSF de 173 para $79 \mathrm{~mL}$, redução de $54,4 \%$, atingindo remodelamento reverso do VE. Por fim, a CF II foi para CF I da NYHA, permanecendo praticamente assintomático.

\section{DISCUSSÃO}

\section{Conceito de super-respondedor à TRC}

Pacientes sem cardiopatia estrutural e com BRE quando comparados aos sem cardiopatia estrutural e sem BRE apresentam, em média, 7\% a menos da FV, considerando-se ambos dentro da faixa da normalidade ${ }^{16}$. Em cerca de três anos, aproximadamente $16 \%$ dos pacientes com coração normal e BRE poderão evoluir com $\mathrm{IC}^{17}$. Essa condição será definida como cardiopatia elétrica primária ${ }^{18-20}$.

Quando atuamos na causa da cardiopatia em questão, de forma geral, temos as melhores taxas de resposta ao tratamento. Acredita-se que esses pacientes são os que apresentam a maior taxa de resposta à TRC - os superrespondedores. É claro que muitos outros pacientes, das mais diversas etiologias de IC, podem evoluir com BRE e beneficiarem-se com a TRC, mas acredita-se que as taxas de resposta sejam mais modestas.

Apesar de não haver consenso em relação ao melhor critério a ser considerado na avaliação da taxa de resposta à TRC, parâmetros clínicos com melhora de pelo menos uma $\mathrm{CF}$ da NYHA, aumento de 10 a $15 \%$ no teste de caminhada de seis minutos, elevação de 10 a $15 \%$ do pico de consumo de oxigênio (VO2 pico) na ergoespirometria, melhora do questionário de vida Minnesota e ecocardiográficos, como a melhora FEVE > 5\% e a redução do VSF maior que 10 a 15\%, são os mais utilizados. Outros critérios, como melhora da pressão arterial e da redução peptídeo natriurético, também podem ser considerados.

Semelhantemente, por não se ter consenso na literatura médica em relação ao conceito do super-respondedor, constam da Tabela 1 alguns dos principais critérios ecocardiográficos utilizados nos grandes estudos sobre o assunto.

Tabela 1. Parâmetros e pontos de cortes usados na definição de super-respondedor.

\begin{tabular}{|c|c|}
\hline Parâmetros & Ponto de corte \\
\hline Função Ventricular ${ }^{19}$ & > 50\% e recuperação funcional \\
\hline Função Ventricular20 & $\begin{array}{l}>2 \times \text { a função de base ou }>45 \% \\
\text { e }>1 \text { Classe funcional NYHA }\end{array}$ \\
\hline Volume Sistólico Final ${ }^{21}$ & Redução > 30\% \\
\hline Volume Diastólico final21 & Redução > 20\% \\
\hline Função Ventricular²1 & Aumento > 10\% \\
\hline Volume Sistólico Final22 & Redução > 30\% \\
\hline Função Ventricular²3 & $\begin{array}{l}>\text { 50\%, redução Volume Sistólico Final } \\
>25 \% \text { e > } 1 \text { Classe funcional NYHA }\end{array}$ \\
\hline Função Ventricular24 & Aumento > 20\% \\
\hline
\end{tabular}

Fonte: Adaptada de Steffel e Ruschitzka25; NYHA = New York Heart Association 


\section{CONCLUSÃO}

A seleção cada vez melhor dos candidatos à TRC, a técnica cirúrgica correta, a programação adequada do dispositivo e o seguimento regular têm contribuído para o aumento da taxa de resposta à TRC. Nesse contexto, destaca-se o conceito do super-respondedor. No caso aqui relatado, elucidamos que quando se sistematiza a abordagem à TRC, aproxima-se cada vez mais dos super-respondedores, atingindo, assim, os melhores resultados que essa modalidade de tratamento pode proporcionar.

\section{CONTRIBUIÇÃO DOS AUTORES}

Todos os autores contribuiram igualmente para este artigo.

\section{REFERÊNCIAS}

1. Rich MW. Heart failure in the 21st century: a cardiogeriatric syndrome. J Gerontol A. 2001;56(2):8896.

2. Krum H, Gilbert RE. Demographics and concomitant disorders in heart failure. Lancet. 2003;362:147-58. https://doi.org/10.1016/S0140-6736(03)13869-X

3. Bui AL, Horwich TB, Fonarow GC. Epidemiology and risk profile of heart failure. Nat Rev Cardiol. 2011;8(1):30-41. https://doi.org/10.1038/nrcardio.2010.165

4. Linde C, Ellenbogen K, Mcalister FA. Cardiac resynchronization therapy (CRT): clinical trial, guidelines, and target populations. Heart Rhytm. 2012;8 Suppl:S3-13. https://doi.org/10.1016/j.hrthm.2012.04.026

5. Salerno HD, Oliveira JC, Melo CS, Lage JS. Evidências atuais para indicação de cardioversores-desfibriladores implantáveis e ressincronizadores cardíacos. In: Melo CS, editor. Temas de marcapasso. 2a ed. São Paulo: Leitura Médica; 2011. p 515-31.

6. Bocchi EA, Marcondes-Braga FA, Bacal F, Ferraz AS, Albuquerque D, Rodrigues DA, et al. Atualização da diretriz brasileira de insuficiência cardíaca crônica 2012. Arq Bras Cardiol. 2012;8 Suppl:S1-33. https://doi. org/10.5935/abc.20120101

7. McMurray JJV, Adamopoulos S, Anker SD, Auricchio A, Bohm M, Dickstein K, et al. ESC Guidelines for the diagnosis and treatment of acute and chronic heart failure. Eur Heart J. 2012;33:1787-847. https://doi. org/10.1093/eurheartj/ehs104

8. European Society of Cardiology (ESC), European Heart Rhythm Association (EHRA), Brignole M, Auricchio A, Baron-Esquivias G, Bordachar P, et al. 2013 ESC guidelines on cardiac pacing and cardiac resynchronization therapy: the task force on cardiac pacing and resynchronization therapy of the European Society of Cardiology (ESC). Developed in collaboration with the European Heart Rhythm Association (EHRA). Europace. 2013;15(8):1070118. https://doi.org/10.1093/europace/eut206

9. Writing Committee Members, Yancy CW, Jessup $M$, Bozkurt B, Butler J, Casey Junior DE, et al. American College of Cardiology Foundation/American Heart Association Task Force on Practice Guidelines. 2013 ACCF/AHA guideline for the management of heart failure: a report of the American College of Cardiology Foundation/American Heart Association Task Force on practice guidelines. Circulation. 2013;128(16):e240-327. https://doi.org/10.1161/CIR.0b013e31829e877610

10. Poole JE, Singh JP, Birgersdotter-Green U. QRS duration or QRS morphology. What really matters in cardiac resynchronization therapy? JACC. 2016;67(9):1104-17. https://doi.org/10.1016/j.jacc.2015.12.039

11. Bleeker GB, Kaandorp TA, Lamb HJ, Boersma E, Steendijk $P$, et al. Effect of posterolateral scar tissue on clinical and echocardiographic improvement after cardiac resynchronization therapy. Circulation. 2006;113:96976. https://doi.org/10.1161/circulationaha.105.543678

12. Ypenburg C, Roes SD, Bleeker GB, Kaandorp TA, Roos A de, Schalij MJ, et al. Effect of total scar burden on contrast-enhanced magnetic resonance imaging on response to cardiac resynchronization therapy Am J Cardiol. 2007;99(5):657-60. https://doi.org/10.1016/j. amjcard.2006.09.115

13. Leyva F, Nisam S, Auricchio A. 20 years of cardiac resynchronization therapy. JACC. 2014;64(10):1047-58. https://doi.org/10.1016/j.jacc.2014.06.1178

14. Lubitz SA, Singh JP. Biventricular pacing: more is better! Eur Heart J. 2015;36(7):407-9. https://doi.org/10.1093/ eurheartj/ehu347

15. Brenyo A, Kutyifa V, Moss AJ, Mathias A, Barsheshet A, Pouleur A-C, et al. Atrioventricular delay programming and the benefit of cardiac resynchronization therapy in MADIT-CRT. Heart Rhyth. 2013;10(8):1101-1248. https:// doi.org/10.1016/j.hrthm.2013.04.013

16. Grines CL, Bashore TM, Boudoulas H, Olson S, Shafer P, Wooley CF. Functional abnormalities in isolated left bundle branch block the effect of interventricular asynchrony. Circulation. 1989;79:845-53.

17. Angheloiu GO, Saul M, Edelman K, Shah H, Mezu UL, Saba S. Predictors of left ventricular function deterioration in patients with left bundle branch block and ejection fraction > 50\%. Congest Heart Fail. 2013;19(4):E1-4. https://doi.org/10.1111/chf.12028

18. Blanc JJ, Fatemi M, Bertault V, Baraket F, Etienne Y. Evaluation of left bundle branch block as a reversible cause of non-ischemic dilated cardiomyopathy with severe heart failure. A new concept of left ventricular dyssynchrony-induced cardiomyopathy. Europace. 2005;7:604-10. https://doi.org/10.1016/j. eupc.2005.06.005 
19. Castellant P, Fatemi M, Orhan E, Etienne Y, Blanc JJ. Patients with non-ischaemic dilated cardiomyopathy and hyper-responders to cardiac resynchronization therapy: characteristics and long-term evolution. Europace. 2009;11(3):350-5. https://doi.org/10.1093/europace/ eup035.

20. Antonio N, Teixeira R, Coelho L, Lourenço C, Monteiro $P$, Ventura $M$, et al. Identification of 'super-responders' to cardiac resynchronization therapy: the importance of symptom duration and left ventricular geometry. Europace. 2009;11(3):343-9. https://doi.org/10.1093/ europace/eup038

21. Steffel J, Milosevic G, Hürlimann A, Krasniqi, Namdar M, Ruschitzka F, et al. Characteristics and long-term outcome of echocardiographic super-responders to cardiac resynchronisation therapy: 'real world' experience from a single tertiary care centre. Heart. 2011;97(20):166874. https://doi.org/ 0.1136/heartjnl-2011-300222
22. Ypenburg C, van Bommel RJ, Borleffs CJ, Bleeker GB, Boersma E, Schalij MJ, et al. Long-term prognosis after cardiac resynchronization therapy is related to the extent of left ventricular reverse remodeling at midterm follow- up. J Am Coll Cardiol. 2009;53(6)483-90. https:// doi.org/10.1016/j.jacc.2008.10.032

23. Reant $P$, Zaroui A, Donal E, Mignot A, Bordachar $P$, Deplagne $A$, et al. Identification and characterization of super-responders after cardiac resynchronization therapy. Am J Cardiol. 2010;105(9):1327-35. https://doi. org/10.1016/j.amjcard.2009.12.058

24. Rickard J, Kumbhani DJ, Popovic Z, Verhaert D, Manne M, Sraow D, et al. Characterization of superresponse to cardiac resynchronization therapy. Heart Rhythm. 2010;(7):885-9. https://doi.org/10.1016/j. hrthm.2010.04.005

25. Steffel J; Ruschitzka F. Superresponse to cardiac resynchronization therapy. Circulation. 2014;130(1):8790. https://doi.org/10.1161/circulationaha.113.006124 